\title{
Non-fragile finite-time guaranteed cost fuzzy control for continuous-time nonlinear systems
}

\author{
Lei Zhang \\ School of Control Science and Engineering, Hebei University of Technology, \\ Tianjin, 300130, China \\ Xiang-Yun Wang \\ College of Computer and Information Engineering, Tianjin Normal University, \\ Tianjin, 300387, China \\ Kun Zhang \\ School of Control Science and Engineering, Hebei University of Technology, \\ Tianjin, 300130, China \\ E-mail: zhangkun@hebut.edu.cn \\ www.hebut.edu.cn
}

Received 24 August 2012

Accepted 4 August 2013

\begin{abstract}
This paper considers the problem of non-fragile finite-time guaranteed cost fuzzy control for continuous-time nonlinear systems. Firstly, the definition on finite-time stability (FTS) for continuous-time nonlinear systems is provided and we give a novel and explicit interpretation for non-fragile finite-time guaranteed cost control. Secondly, sufficient conditions for the existence of state feedback controller are derived in terms of linear matrix inequities (LMIs), which guarantee the requirements of the provided performance criterion. The related optimization problem is also offered to minimize the guaranteed cost performance bound. Finally, an illustrative example is presented to show the validity of the proposed scheme.
\end{abstract}

Keywords: Finite-time stability, Guaranteed cost , Fuzzy control, Linear matrix inequality

\section{Introduction}

Since there exist many cases where the state values are restrained, we generally need to ensure that these state values are allowable. In order to deal with these cases, the concept of finite-time stability (FTS) (or short-time stability) can be introduced into our eyeshot. As illuminated in [1], a system is said to be finite-time stable if, once we fix a time-interval, its state does not exceed certain bound during this time-interval. Some early results on FTS can be found in $[2,3]$. More recently the problem of finite-time control for both continuous-time and discrete-time linear systems has been investigated in $[1,4,5]$ via linear matrix inequities (LMIs) technique. In the existing results, FTS is mainly associated with linear systems. How to solve the FTS problem for nonlinear systems is still a difficult problem. Recently, Takagi-Sugeno (T-S) fuzzy model proposed in [6] is widely applied in various industrial control 
fields because of its simple structure with local dynamics. The typical approach named as parallel distributed compensation (PDC) [7] is also developed to design the fuzzy controllers. Moreover, besides the stabilization problem, the guaranteed cost fuzzy control was extendedly investigated to stabilize the controlled systems while providing an upper bound on a given performance index by many scholars in the last decade, such as [8 11].

A concept for non-fragile control is proposed for the following problem: how to design a feedback control that will be insensitive to some error in gains of feedback control [12]. The states of a system are not always measurable or have practical sense in many control systems and applications. And all parameters in the control have some variations due to additive unknown noise and environmental influence. Recently, some related results are also discussed by many scholars [13-15]. However these results have not considered finite-time control case.

In this paper, non-fragile controls are considered, which contains some uncertainties in control gains. And we propose a non-fragile finite-time guaranteed cost fuzzy control scheme for continuous-time nonlinear systems. Its objective is to find a suitable state feedback controller such that the provided performance criterion is satisfied. As far as we know, up to date, the nonfragile finite-time guaranteed cost control for continuous-time nonlinear systems has not been considered in the existing results.

The remainder of this paper is organized as follows: Basic problem formulation is introduced in Section 2. The non-fragile finite-time guaranteed cost controller via state feedback is designed in Section 3. Section 4 provides an illustrative example to demonstrate the effectiveness of the proposed scheme. Finally, concluding remarks are made in Section 5 .

In the following sections, the identity matrices and zero matrices are denoted by $I$ and 0 , respectively. $X^{T}$ denotes the transpose of matrix $X . R^{n}$ denotes the n-dimensional Euclidean space. $R_{+}$denotes the positive real number. The notation * always denotes the symmetric block in one symmetric matrix. The standard notation $>(<)$ is used to denote the positive (negative)definite ordering of matrices. Inequality $X>Y$ shows that the matrix $X-Y$ is positive definite. $\lambda_{\max }(\cdot)$ and $\lambda_{\min }(\cdot)$ indicate the maximum and minimum eigenvalues of the matrix, respectively.

\section{Problem formulation}

Consider a general class of continuous-time nonlinear systems which can be represented by the following T-S fuzzy dynamic model.

\section{Plant Rule $i$}

$$
\begin{aligned}
& \text { IF } \theta_{1}(t) \text { is } N_{i 1}, \cdots, \text { and } \theta_{p}(t) \text { is } N_{i p} \\
& \text { THEN } \dot{x}(t)=A_{i} x(t)+B_{i} u(t)
\end{aligned}
$$

where $i=1,2, \cdots, r, r$ is the number of fuzzy rules, $x(t) \in R^{n}$ is the state vector, $u(t) \in R^{m}$ is the control input, $A_{i} \in R^{n \times n}$ and $B_{i} \in R^{n \times m}$ are known constant matrices of the $i-t h$ subsystem, $\theta_{1}(t), \theta_{2}(t), \cdots, \theta_{p}(t)$ are the premise variables, $N_{i j}$ is the fuzzy set $(j=1,2, \cdots, p)$. By using singleton fuzzifier, product inference and center-average defuzzifer, the fuzzy dynamic model is expressed by

$$
\dot{x}(t)=\sum_{i=1}^{r} h_{i}(\theta(t))\left[A_{i} x(t)+B_{i} u(t)\right]
$$

$$
\text { where, } h_{i}(\theta(t))=\mu_{i}(\theta(t)) / \sum_{i=1}^{r} \mu_{i}(\theta(t))
$$

$$
\mu_{i}(\theta(t))=\prod_{j=1}^{p} N_{i j}\left(\theta_{j}(t)\right) \text { and } N_{i j}\left(\theta_{j}(t)\right) \text { is the }
$$
grade of membership of $\theta_{j}(t)$ in $N_{i j}$. It is assumed that $\mu_{i}(\theta(t)) \geq 0$ and $\sum_{i=1}^{r} \mu_{i}(\theta(t))>0$ for all t. Then, we can see that $h_{i}(\theta(t)) \geq 0$ and $\sum_{i=1}^{r} h_{i}(\theta(t))=1$.

According to the conventional PDC concept, we design the following fuzzy controller via state feedback:

\section{Controller Rule $i$}

$$
\begin{aligned}
& \text { IF } \theta_{1}(t) \text { is } N_{i 1}, \cdots, \text { and } \theta_{p}(t) \text { is } N_{i p} \\
& \text { THEN } u(t)=K_{i} x(t)
\end{aligned}
$$

where $i=1,2, \cdots, r, r$ is the number of controller rules, $K_{i} \in R^{n \times m}$ is the controller gain matrix. Thus, the fuzzy controller can be expressed as follows:

$$
u(t)=\sum_{i=1}^{r} h_{i}(\theta(t)) K_{i} x(t)
$$

The closed-loop system (1) can be reconstructed under the fuzzy controller (2) as follows:

$$
\dot{x}(t)=\sum_{i=1}^{r} h_{i}(\theta(t))\left[A_{i} x(t)+B_{i} u(t)\right]=A x(t)
$$


where $A=\sum_{i=1}^{r} \sum_{j=1}^{r} h_{i}(\theta(t)) h_{j}(\theta(t))\left[A_{i}+B_{i} K_{j}\right]$.

Next we will provide a novel concept on FTS for continuous-time nonlinear systems. It is formalized through the following definition. Similar description can be found in [1].

Definition 1: The closed-loop continuous-time nonlinear system (3) is said to be finite-time stable (FTS) with respect to $\left(c_{1}, c_{2}, T, R_{C}\right)$ where $0<c_{1}<c_{2}$, $T \in R_{+}$and $R_{C}>0$ if

$$
x^{T}(0) R_{C} x(0) \leq c_{1} \Rightarrow x^{T}(t) R_{C} x(t) \leq c_{2} \quad \forall t \in(0, T]
$$

Remark 1: Asymptotic stability and FTS are independent concepts each other as it was described in [1]. In certain cases, asymptotic stability can be also considered as an additional requirement while restricting our attention on a finite-time interval.

Generally, matrices $A_{i}$ and $B_{i}$ can be identified through available data. If there exist the approximation errors between the original nonlinear system and T-S fuzzy model, we can utilize a robust stabilization technique for dealing with the errors. In this paper, nonfragile control for the practical implement is considered as follows:

$$
\dot{x}(t)=\sum_{i=1}^{r} \sum_{j=1}^{r} h_{i}(\theta(t)) h_{j}(\theta(t))\left[A_{i}+B_{i}\left(K_{j}+\Delta K_{j}\right)\right] x(t)
$$

where $\Delta K_{j}(j=1,2, \cdots, r)$ are perturbed matrices. Two types of perturbations are considered:

Type 1: Perturbations are independent on matrices $K_{j}(j=1,2, \cdots, r)$ :

$$
\Delta K_{j}=M_{j} F_{j}(t) N_{j}
$$

Type 2: Perturbations are dependent on matrices $K_{j}(j=1,2, \cdots, r)$ :

$$
\Delta K_{j}=M_{j} F_{j}(t) N_{j} K_{j}
$$

In the above two types of perturbations, matrices $M_{j}$ and $N_{j}$ are known with appropriate dimension. Timevarying matrices $F_{j}(t)$ are unknown continuous functions and satisfying

$$
F_{j}^{T}(t) F_{j}(t) \leq I \quad t \geq 0
$$

Furthermore, for (4), we provide the following cost function associated with the above definition:

$$
J=\int_{0}^{T}\left[x^{T}(t) Q_{1} x(t)+u^{T}(t) Q_{2} u(t)\right] d t
$$

where $Q_{1}>0$ and $Q_{2}>0$ are given weighting matrices or given positive scalars. The following novel and explicit interpretation for non-fragile finite-time guaranteed cost control is given by:
Definition 2: For (5), if there exists a fuzzy control law (4) and a scalar $\Xi$ such that the closed-loop system is finite-time stable and the value of the cost function (7) satisfies $J<\Xi$, then $\Xi$ is said to be a guaranteed cost bound and the designed control law (4) is said to be a finite-time guaranteed cost fuzzy control law.

Lemma 1 [16]: Let $U, V, W$ and $M$ be real matrices of appropriate dimensions with $M$ satisfying $M=M^{T}$, then $M+U V W+W^{T} V^{T} U^{T}<0$ for all $V^{T} V \leq I$, if and only if there exists a scalar $\varepsilon>0$ such that

$M+\varepsilon^{-1} U U^{T}+\varepsilon W^{T} W<0$
Lemma 2 [17]: For a given matrix with $S=\left[\begin{array}{ll}S_{11} & S_{12} \\ S_{12}^{T} & S_{22}\end{array}\right]$ $S_{11}=S_{11}^{T}$ and $S_{22}=S_{22}^{T}$, then the following conditions are equivalent:

(1) $S<0$

(2) $S_{22}<0, S_{11}-S_{12} S_{22}^{-1} S_{12}^{T}<0$

Lemma 3: For any given $X, Y \in R^{n \times 1}$, the matrix inequality $X^{T} Y+Y^{T} X \leq X^{T} X+Y^{T} Y \quad$ always is satisfied.

\section{Main results}

In this section, the problem of non-fragile finite-time guaranteed cost fuzzy control via state feedback is studied according to (4). Some results are provided as follows.

Theorem 1: If there exist a scalar $\alpha \geq 0$ and $\varepsilon>0$, the symmetric positive definite matrix $Q \in R^{n \times n}$ and matrices $\left(W_{j}, M_{j}, N_{j}\right)$ with the appropriate dimensions for $1 \leq i, j \leq r$ such that the conditions (8-1) (9) are satisfied, the continuous-time nonlinear system (4) with perturbations (5) is FTS with respect to $\left(c_{1}, c_{2}, T, R_{C}\right)$ where $0<c_{1}<c_{2}, T \in R_{+}, R_{C}>0$ and has the guaranteed cost bound $\Xi$. Moreover, the controller gain matrix $K_{j}=W_{j} \tilde{Q}^{-1}$.

$$
\left\{\begin{array}{l}
F_{j}^{T}(t) F_{j}(t) \leq I \\
F_{j}^{T}(t) M_{j}^{T} Q_{2}(t) M_{j}(t) F_{j}(t) \leq 2 I
\end{array} \quad(t \geq 0,1 \leq j \leq r)\right.
$$

$\left[\begin{array}{ccccccccc}\Psi_{i i} & \tilde{Q} & W_{i}^{T} & B_{i} M_{i} & \varepsilon\left(N_{i} \tilde{Q}\right)^{T} & W_{i}^{T} & \varepsilon\left(N_{i} \tilde{Q}\right)^{T} & \left(N_{i} \tilde{Q}\right)^{T} & \varepsilon\left(N_{i} \tilde{Q}\right)^{T} \\ \tilde{Q} & -Q_{1}^{-1} & 0 & 0 & 0 & 0 & 0 & 0 & 0 \\ W_{i} & 0 & -Q_{2}^{-1} & 0 & 0 & 0 & 0 & 0 & 0 \\ \left(B_{i} M_{i}\right)^{T} & 0 & 0 & -\varepsilon I & 0 & 0 & 0 & 0 & 0 \\ \varepsilon N_{i} \tilde{Q}_{i} & 0 & 0 & 0 & -\varepsilon I & 0 & 0 & 0 & 0 \\ W_{i} & 0 & 0 & 0 & 0 & -\varepsilon I & 0 & 0 & 0 \\ \varepsilon N_{i} \tilde{Q} & 0 & 0 & 0 & 0 & 0 & -\varepsilon I & 0 & 0 \\ N_{i} \tilde{Q} & 0 & 0 & 0 & 0 & 0 & 0 & -\varepsilon I & 0 \\ \varepsilon N_{i} \tilde{Q} & 0 & 0 & 0 & 0 & 0 & 0 & 0 & -\varepsilon I\end{array}\right]<0 \quad 1 \leq i \leq r$




$$
\frac{c_{1}}{\lambda_{\min }(Q)}<\frac{c_{2} e^{-\alpha T}}{\lambda_{\max }(Q)}
$$

where $\bar{\psi}_{i i}=\tilde{Q} A_{i}^{T}+A_{i} \tilde{Q}+W_{i}^{T} B_{i}^{T}+B_{i} W_{i}-\alpha \tilde{Q}$, $\bar{\Omega}_{u_{i}}=\tilde{Q}\left(A_{i}+B_{i} K_{j}\right)^{T}+\left(A_{i}+B_{i} K_{j}\right) \tilde{Q}+\tilde{Q}\left(A_{j}+B_{j} K_{i}\right)^{T}+\left(A_{j}+B_{j} K_{i}\right) \tilde{Q}-2 \alpha \tilde{Q}$ and $\tilde{Q}=R_{C}^{-1 / 2} Q R_{C}^{-1 / 2}$. The guaranteed cost bound is described by:

$$
\Xi=\lambda_{\max }\left(Q^{-1}\right) c_{1} e^{\alpha T}
$$

PROOF: Let $V(x(t))=x^{T}(t) \tilde{Q}^{-1} x(t)$ and assume the time derivative of $V(x(t))$ along the solution of system (4) to satisfy the following condition for all $\forall t \in(0, T]$

$$
\dot{V}(x(t))<\alpha V(x(t))
$$

Dividing both sides of (10) by $V(x(t))$ and integrating from 0 to $t$, with $t \in(0, T]$, we obtain

$$
\ln \frac{V(x(t))}{V(x(0))}<\alpha t
$$

From (11), we can obtain the following inequality:

$$
x^{T}(t) R_{C}^{1 / 2} Q^{-1} R_{C}^{1 / 2} x(t)<x^{T}(0) R_{C}^{1 / 2} Q^{-1} R_{C}^{1 / 2} x(0) e^{\alpha t}
$$

Now we have

$$
\begin{aligned}
x^{T}(t) R_{C}^{1 / 2} Q^{-1} R_{C}^{1 / 2} x(t) & \geq \lambda_{\min }\left(Q^{-1}\right) x^{T}(t) R_{C} x(t) \\
x^{T}(0) R_{C}^{1 / 2} Q^{-1} R_{C}^{1 / 2} x(0) & \leq \lambda_{\max }\left(Q^{-1}\right) x^{T}(0) R_{C} x(0) \\
& \leq \lambda_{\max }\left(Q^{-1}\right) c_{1}
\end{aligned}
$$

Combining (12) (14), we have

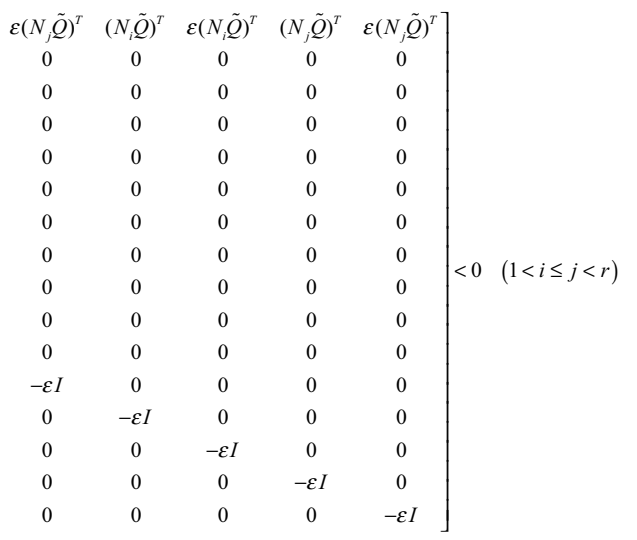

$$
\begin{aligned}
x^{T}(t) R_{C} x(t) & <\frac{\lambda_{\max }\left(Q^{-1}\right) c_{1} e^{\alpha t}}{\lambda_{\min }\left(Q^{-1}\right)} \\
& =\lambda_{\max }(Q) e^{\alpha t} \frac{\lambda c_{1}}{\lambda_{\min }(Q)} \\
& \leq \lambda_{\max }(Q) e^{\alpha T} \frac{\lambda c_{1}}{\lambda_{\min }(Q)}
\end{aligned}
$$

Consider $x^{T}(t) R_{C} x(t)<c_{2}$ for all $t \in(0, T]$ in Definition 1. The condition (9) in Theorem 1 is obtained. From condition (8-1), condition (8-2), Lemma 1 and Lemma 2, we have

$$
\begin{aligned}
& \psi_{i i}=\left(A_{i}+B_{i}\left(K_{i}+\Delta K_{i}\right)\right)^{T} \tilde{Q}^{-1}+\tilde{Q}^{-1}\left(A_{i}+B_{i}\left(K_{i}+\Delta K_{i}\right)\right) \\
& -\alpha \tilde{Q}^{-1}+Q_{1}+\left(K_{i}+\Delta K_{i}\right)^{T} Q_{2}\left(K_{i}+\Delta K_{i}\right)<0 \quad(1 \leq i \leq r)
\end{aligned}
$$

From condition (8-1),condition (8-3), Lemma 1 and Lemma 2, we have

$$
\begin{aligned}
& \Omega_{j j}=\left(A_{i}+B_{i}\left(K_{j}+\Delta K_{j}\right)\right)^{T} \tilde{Q}^{-1}+\tilde{Q}^{-1}\left(A_{i}+B_{i}\left(K_{j}+\Delta K_{j}\right)\right) \\
& +\left(A_{j}+B_{j}\left(K_{i}+\Delta K_{i}\right)\right)^{T} \tilde{Q}^{-1}+\tilde{Q}^{-1}\left(A_{j}+B_{j}\left(K_{i}+\Delta K_{i}\right)\right)-20 \tilde{Q}^{-1} \\
& +2 Q_{+}+\left(K_{i}+\Delta K_{i}\right)^{T} Q_{2}\left(K_{i}+\Delta K_{i}\right)+\left(K_{j}+\Delta K_{j}\right)^{T} Q_{2}\left(K_{j}+\Delta K_{j}\right)<0 \quad(1 \leq i<j \leq r)
\end{aligned}
$$

Denoting $\quad \Delta V \square \dot{V}(x(t))-\alpha V(x(t)) \quad$ and utilizing Lemma 2, we can obtain the following result:

$\left.\Delta V=\sum_{i=1}^{r} \sum_{j=1}^{r} h_{1}(\theta(t)) h_{j}(\theta(t))\right)^{T}(t)\left[\left(A_{i}+B_{i}\left(K_{j}+\Delta K_{j}\right)\right)^{T} \tilde{Q}^{-1}+\tilde{Q}^{-1}\left(A_{i}+B_{i}\left(K_{j}+\Delta K_{j}\right)\right)-\alpha \tilde{Q}^{-1}\right] x(t)$ $\leq \sum_{i=1}^{r} h_{i}(\theta(t)) h_{i}(\theta(t)) x^{T}(t) \psi_{i i} x(t)+\sum_{i=1}^{r-1} \sum_{j>i}^{r} h_{i}(\theta(t)) h_{j}(\theta(t)) x^{T}(t) \Omega_{i j} x(t)$

$-\sum_{i=1 j=1}^{r} \sum_{i=1}^{r} h_{i}(\theta(t)) h_{j}(\theta(t)) x^{T}(t)\left(Q+\left(K_{i}+\Delta K_{i}\right)^{T} Q_{(}\left(K_{i}+\Delta K_{i}\right)\right) x(t)$

From the above derivation, we can obtain that if $\psi_{i i}<0$ and $\Omega_{i j}<0$ hold, then $\Delta V<0$ for any 
nonzero $x(t)$, i.e., (10) is true. After integrating actions from 0 to $T$, the following result is obtained:

$$
-\lambda_{\max }\left(Q^{-1}\right) c_{1} e^{\alpha T} \leq \int_{0}^{T} \Delta V(x(t)) d t \leq-J
$$

Moreover, $J<\lambda_{\max }\left(Q^{-1}\right) c_{1} e^{\alpha T}$ can be obtained by considering definition 1 . It is easy to see that the guaranteed cost function satisfies $J<\Xi=\lambda_{\max }\left(Q^{-1}\right) c_{1} e^{\alpha T}$.

The proof is completed.

Remark 2: If the conditions (8-1) (9) in Theorem 1 is true for $\alpha=0$, then it can be easily seen that Lyaponuv asymptotic stability (LAS) for the continuous-time nonlinear system (4) will be guaranteed.

Consequently, the result about the feasible probl em of LMIs is provided as follows.

Corollary 1: If there exist a scalar $\alpha \geq 0$ and $\varepsilon>0$, the symmetric positive definite matrix $Q \in R^{n \times n}$ and matrices $\left(W_{j}, M_{j}, N_{j}\right)$ with the appropriate dimensions for $1 \leq i, j \leq r$ such that the conditions (8-1) (8-3) and (15) are satisfied, the continuous-time nonlinear system (4) with perturbations (5) is FTS with respect to $\left(c_{1}, c_{2}, T, R_{C}\right)$ where $0<c_{1}<c_{2}, T \in R_{+}, R_{C}>0$ and has the guaranteed cost bound $\Xi$. Moreover, the controller gain matrix $K_{j}=W_{j} \tilde{Q}^{-1}$.

$$
\frac{c_{1}}{c_{2}} e^{\alpha T} I<Q<I
$$

where $\bar{\psi}_{i i}=\tilde{Q} A_{i}^{T}+A_{i} \tilde{Q}+W_{i}^{T} B_{i}^{T}+B_{i} W_{i}-\alpha \tilde{Q}$,

$\bar{\Omega}_{i i}=\tilde{Q}\left(A_{i}+B_{i} K_{j}\right)^{T}+\left(A_{i}+B_{i} K_{j}\right) \tilde{Q}+\tilde{Q}\left(A_{j}+B_{j} K_{i}\right)^{T}+\left(A_{j}+B_{j} K_{i}\right) \tilde{Q}-20 \tilde{Q}$ and $\tilde{Q}=R_{C}^{-1 / 2} Q R_{C}^{-1 / 2}$.

PROOF: Since the conditions $(8-1) \sim(8-3)$ in Theorem 1 has already been described via LMI, so we only need a LMI displacement for the condition (9) in Theorem 1. Utilizing the result in [2], if the LMI $\frac{c_{1}}{c_{2}} e^{\alpha T} I<Q<I$ holds, the condition (9) in Theorem 1 will be true. The proof is completed.

Theorem 2: If there exist a scalar $\alpha \geq 0$ and $\varepsilon>0$, the symmetric positive definite matrix $Q \in R^{n \times n}$ and matrices $\left(W_{j}, M_{j}, N_{j}\right)$ with the appropriate dimensions for $1 \leq i, j \leq r$ such that the conditions (16-1) (17) are satisfied, the continuous-time nonlinear system (4) with perturbations (6) is FTS with respect to $\left(c_{1}, c_{2}, T, R_{C}\right)$ where $0<c_{1}<c_{2}, T \in R_{+}, R_{C}>0$ and has the guaranteed cost bound $\Xi$. Moreover, the controller gain matrix $K_{j}=W_{j} \tilde{Q}^{-1}$.

$$
\left\{\begin{array}{l}
F_{j}^{T}(t) F_{j}(t) \leq I \\
F_{j}^{T}(t) M_{j}^{T} K_{j}^{T} Q_{2}(t) K_{j} M_{j}(t) F_{j}(t) \leq 2 I
\end{array}(t \geq 0,1 \leq j \leq r)\right.
$$




$$
\frac{c_{1}}{\lambda_{\min }(Q)}<\frac{c_{2} e^{-\alpha T}}{\lambda_{\max }(Q)}
$$

where $\bar{\psi}_{i i}=\tilde{Q} A_{i}^{T}+A_{i} \tilde{Q}+W_{i}^{T} B_{i}^{T}+B_{i} W_{i}-\alpha \tilde{Q}$,

$$
\bar{\Omega}_{i i}=\tilde{Q}\left(A_{i}+B_{i} K_{j}\right)^{T}+\left(A_{i}+B_{i} K_{j}\right) \tilde{Q}+\tilde{Q}\left(A_{j}+B_{j} K_{i}\right)^{T}
$$$$
+\left(A_{j}+B_{j} K_{i}\right) \tilde{Q}-2 \alpha \tilde{Q}
$$

and $\tilde{Q}=R_{C}^{-1 / 2} Q R_{C}^{-1 / 2}$. The guaranteed cost bound is described by:

$$
\Xi=\lambda_{\max }\left(Q^{-1}\right) c_{1} e^{\alpha T}
$$

Corollary 2: If there exist a scalar $\alpha \geq 0$ and $\varepsilon>0$, the symmetric positive definite matrix $Q \in R^{n \times n}$ and matrices $\left(W_{j}, M_{j}, N_{j}\right)$ with the appropriate dimensions for $1 \leq i, j \leq r$ such that the conditions (16-1) (16-3)and (18) are satisfied, the continuous-time nonlinear system (4) with perturbations (6) is FTS with respect to $\left(c_{1}, c_{2}, T, R_{C}\right)$ where $0<c_{1}<c_{2}, T \in R_{+}, R_{C}>0$ and has the guaranteed cost bound $\Xi$. Moreover, the controller gain matrix $K_{j}=W_{j} \tilde{Q}^{-1}$.

$$
\frac{c_{1}}{c_{2}} e^{\alpha T} I<Q<I
$$

where $\bar{\psi}_{i i}=\tilde{Q} A_{i}^{T}+A_{i} \tilde{Q}+W_{i}^{T} B_{i}^{T}+B_{i} W_{i}-\alpha \tilde{Q}$,

$$
\bar{\Omega}_{i i}=\tilde{Q}\left(A_{i}+B_{i} K_{j}\right)^{T}+\left(A_{i}+B_{i} K_{j}\right) \tilde{Q}+\tilde{Q}\left(A_{j}+B_{j} K_{i}\right)^{T}
$$

$+\left(A_{j}+B_{j} K_{i}\right) \tilde{Q}-2 \alpha \tilde{Q}$

and $\tilde{Q}=R_{C}^{-1 / 2} Q R_{C}^{-1 / 2}$.

The proof of Theorem 2 and Corollary 2 are similar to Theorem 1 and Corollary 1, respectively. The following optimization problems can be presented:

Problem 1: $\min \Xi$ s.t. the conditions (8-1) (8-3) and (15) are satisfied.

Problem 2: $\min \Xi$ s.t. the conditions (16-1) (16-3)and (18) are satisfied.

\section{An illuminated example}

In this section, we will design a finite-time guaranteed cost fuzzy controller for the following continuous-time non linear system as in [18].

Lorenz Equation with Input Term:

$$
\left\{\begin{array}{l}
\dot{x}_{1}(t)=-a x_{1}(t)+a x_{2}(t)+u(t) \\
\dot{x}_{2}(t)=c x_{1}(t)-x_{2}(t)-x_{1}(t) x_{3}(t) \\
\dot{x}_{3}(t)=x_{1}(t) x_{2}(t)-b x_{3}(t)
\end{array}\right.
$$

where $a, b$ and $c$ are constants, $u(t)$ is the control input. Assume that $x_{1}(t) \in[-d, d]$ and $d>0$, Then, the following fuzzy model will exactly represents the nonlinear equation under $x_{1}(t) \in[-d, d]$.

Rule 1: IF $x_{1}(t)$ is $M_{1}\left(x_{1}(t)\right)$, THEN $\dot{x}(t)=A_{1} x(t)+B u(t)$

Rule 2: IF $x_{1}(t)$ is $M_{2}\left(x_{1}(t)\right)$, THEN $\dot{x}(t)=A_{2} x(t)+B u(t)$

$$
\text { where } \quad x(t)=\left[\begin{array}{lll}
x_{1}(t) & x_{2}(t) & x_{3}(t)
\end{array}\right]^{T}
$$

$A_{1}=\left[\begin{array}{ccc}-a & a & 0 \\ c & -1 & -d \\ 0 & d & -b\end{array}\right], A_{2}=\left[\begin{array}{ccc}-a & a & 0 \\ c & -1 & d \\ 0 & -d & -b\end{array}\right], B=\left[\begin{array}{l}1 \\ 0 \\ 0\end{array}\right]$,

$M_{1}\left(x_{1}(t)\right)=\frac{1}{2}\left(1+\frac{x_{1}(t)}{d}\right), M_{2}\left(x_{1}(t)\right)=\frac{1}{2}\left(1-\frac{x_{1}(t)}{d}\right)$.

In this paper, $a=10, b=8 / 3, c=28$ and $d=30$. Moreover, $Q_{1}$ and $Q_{2}$ in the cost function (7) are given as $Q_{1}=\operatorname{diag}[1,1,1]$ and $Q_{2}=1$.

Case 1: Setting $c_{1}=1, c_{2}=5, R_{C}=I, \alpha=0.8$, $\varepsilon=0.1 \quad, \quad T=10 \quad, \quad M_{1}=M_{2}=\left[\begin{array}{ll}1 & 0\end{array}\right]$, $N_{1}=N_{2}=\left[\begin{array}{lll}1 & 1 & 1\end{array}\right] \quad, \quad F_{1}(t)=F_{2}(t)=\left[\begin{array}{c}\sin (t) \\ 0\end{array}\right] \quad$ and $\Delta K_{j}=M_{j} F_{j}(t) N_{j}(j=1,2)$. The minimum of the guaranteed cost bound and the controller gain matrices are obtained as follows:

$\Xi=296.609078$

$K_{1}=\left[\begin{array}{lll}-25.0072 & -10.4628 & 0.5061\end{array}\right]$

$K_{2}=\left[\begin{array}{lll}-25.0126 & -10.4651 & -0.6125\end{array}\right]$

When the initial condition is chosen as $x(0)=\left[\begin{array}{lll}1 & 0 & 0\end{array}\right]^{T}$, the response of state $x$ is showed in Fig. 1.

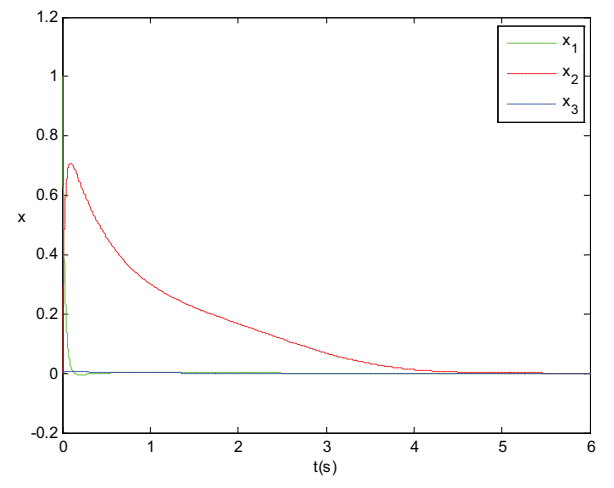

Fig. 1 Response of state $x$ in case 1 
Case 2: Setting $c_{1}=1, c_{2}=5, R_{C}=I, \alpha=0.3$, $\varepsilon=0.1, T=10, M_{1}=M_{2}=\left[\begin{array}{ll}1 & 0\end{array}\right], \quad N_{1}=N_{2}=1$, $F_{1}(t)=F_{2}(t)=\left[\begin{array}{c}\sin (t) \\ 0\end{array}\right]$ and $\Delta K_{j}=M_{j} F_{j}(t) N_{j} K_{j}(j=1,2)$. The minimum of the guaranteed cost bound and the controller gain matrices are obtained as follows:

$\Xi=645.5513$

$K_{1}=\left[\begin{array}{lll}-31.6569 & -13.4660 & 0.4271\end{array}\right]$

$K_{2}=\left[\begin{array}{lll}-31.6569 & -13.4660 & 0.4271\end{array}\right]$

When the initial condition is chosen as $x(0)=\left[\begin{array}{lll}1 & 0 & 0\end{array}\right]^{T}$, the response of state $x$ is showed in Fig. 2.

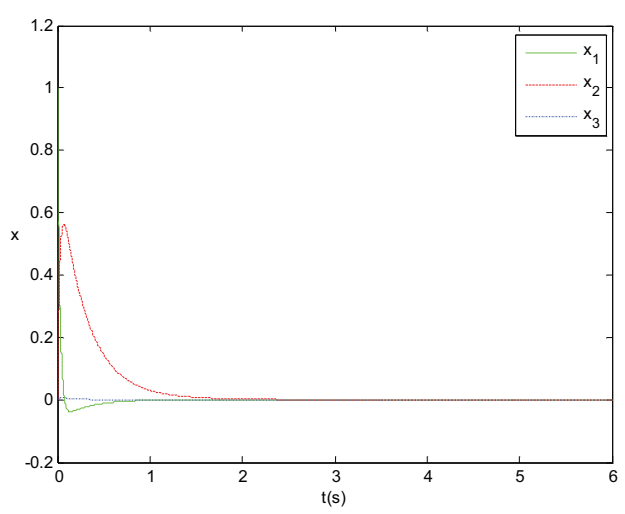

Fig. 2 Response of state $x$ in case 2

\section{Conclusions}

In this study, a non-fragile finite-time guaranteed cost fuzzy control scheme for continuous-time nonlinear systems has been investigated. The whole closed-loop system satisfies the requirements of the provided performance criterion. An illustrative example is given to illustrate the effectiveness of the proposed scheme.

\section{References}

1. F. Amato, M. Ariola, P. Dorato, Finite-time control of linear systems subject to parametric uncertainties and disturbances, Automatica, 2001, 37(9): 1459-1463.

2. P. Dorato, Short time stability in linear time-varying systems, In: Proceedings of the IRE International Convention Record Part 4, 1961: 83-87.
3. L. Weiss, E. F. Infante, Finite time stability under perturbing forces and on product spaces, IEEE Trans. Automatic Control, 1967, 12(1): 54-59.

4. F. Amato, M. Ariola, Finite-time control of discretetime linear systems, IEEE Trans. Automatic Control, 2005, 50(5): 724-729.

5. F. Amato, M. Ariola, C. Cosentino, Finite-time stabilization via dynamic output feedback, Automatica, 2006, 42(2): 337-342.

6. T. Takagi, M. Sugeno, Fuzzy identification of systems and its applications to modeling and control, IEEE Trans. Systems, Man, and Cybernetics, 1985, 15(1): 116-132.

7. H. O. Wang, K. Tanaka, M. F Griffin, An approach to fuzzy control of nonlinear systems: stability and design issues, IEEE Trans. Fuzzy Systems, 1996, 4(1): 4-23.

8. B. Chen, X. Liu, S. Tong, Adaptive fuzzy output tracking control of MIMO nonlinear uncertain systems, IEEE Trans. Fuzzy Systems, 2007, 15(2): 287-300.

9. B. Chen, X. Liu, Fuzzy guaranteed cost control for nonlinear systems with time-varying delay, IEEE Transactions on Fuzzy Systems, 2005, 13(2): 238-249.

10. X. P. Guan, C. L. Chen, Delay-dependent guaranteed cost control for T-S fuzzy systems with time delays, IEEE Transactions on Fuzzy Systems, 2004, 12(2): 236-249.

11. X. Jiang, Q. L. Han, On guaranteed cost fuzzy control for nonlinear systems with interval time-varying delay, IET Control Theory and Application, 2007, 1(6): 1700-1710.

12. P. Dorato, Non-fragile controller design: an overview, In: American Control Conference, 1998: 2829-2831.

13. H. Hu, B. Jiang, $\mathrm{H}$. Yang, Non-fragile $\mathrm{H}_{2}$ reliable control for switched linear systems with actuator faults, Signal Processing, 2013, 93(7): 1804-1812.

14. L. Liu, Z. Fu, X. Cai, X. Song, Non-fragile sliding mode control of discrete singular systems, Communications in Nonlinear Science and Numerical Simulation, 2013, 18(3): 735-743.

15. J. Ren, Q. Zhang, Non-fragile PD state $\mathrm{H}_{\infty}$ control for a class of uncertain descriptor systems, Applied Mathematics and Computation, 2012, 218(17): 88068815.

16. L. Boyd, El. Ghaoui, E. Feron, V. Balakrishnan, Linear matrix inequalities in system and control theory, SIAM, 1994.

17. V. Singh, Robust stability of cellular neural networks with delay: linear matrix inequality approach. In: IEE Proc Control Theory Application, 2004(151): 125-129.

18. K. Tanaka, T. Ikeda, H. O. Wang, A unified approach to controlling chaos via an LMI-based fuzzy control system design, IEEE Trans. Circuits and Systems I: Fundamental Theory and Applications, 1998, 45(10): 1021-1040. 